\title{
Population genetic variation of serotonin transporter gene (SLC6A4), associated with neurophysiological development
}

\author{
Shyamala Hande \\ Melaka Manipal Medical College \\ Manipal, India \\ shyamala.hande@manipal.edu \\ Komalroop Kaur Sahote \\ Melaka Manipal Medical College \\ Manipal, India \\ Nirosha Dev \\ Melaka Manipal Medical College \\ Manipal, India
}

\author{
Ting Pei Erl \\ Melaka Manipal Medical College \\ Manipal, India \\ Kovindraam Ramakrishna \\ Melaka Manipal Medical College \\ Manipal, India \\ Renuka Ravidhran \\ Yenepoya Research Centre, Yenepoya \\ (Deemed to be University), Mangalore \\ Karnataka, India
}

\author{
Swathy M. Krishna \\ Yenepoya Research Centre, Yenepoya \\ (Deemed to be University), Mangalore \\ Karnataka, India \\ Ranajit Das \\ Melaka Manipal Medical College \\ Manipal, India
}

\begin{abstract}
The serotonergic pathway, including the neurotransmitter serotonin and its cognate transporter protein (5-HTT), is known to influence a wide range of individual behavioral traits and cognitive abilities. The serotonin transporter 5-HTT is encoded by a single gene SLC6A4. Polymorphisms in SLC6A4 has been associated with a wide variety of neurological and psychiatric disorders including increased risk of post-traumatic stress disorder, higher likelihood for depression, obsessive-compulsive disorder (OCD), increased hostility and criminal behavior. All diseases associated with $S L C 6 A 4$ are complex disorders, caused by the interplay of various genetic and environmental factors. It has been shown that genes associated with complex diseases exhibit strong signatures of purifying selection compared to non-disease-causing genes. Purifying selection, in several cases, is associated with background selection, where alongside the deleterious alleles, the linked neutral (or even slightly beneficial) alleles are purged away from the genome. The majority of the genetic variations are rare, arose recently, and are highly ancestryspecific. In this project we aimed to investigate the population genetic variation of the serotonin transporter gene (SLC6A4), focusing on the Single Nucleotide Polymorphisms (SNPs). We further aimed to assess whether any modern-day human population show strong signature of natural selection for $S L C 6 A 4$ and has significantly differentiated from the rest of the world for this gene, employing 2504 individuals around the globe available in 1000 Genome project Phase III data.
\end{abstract}

Keywords - Polymorphism in SLC6A4, Ancestry specific variation of natural selection, Signatures of natural selection

\section{Methods}

Data collection The lists of SNPs, present in SLC6A4, were curated from SNPedia and UCSC genome browser. 25 SLC6A4 SNPs, associated with several neuropsychological disorders, were obtained from SNPedia and all 147 annotated SNPs of SLC6A4 were obtained from UCSC genome browser. Whole genome data in the form of VCF files were obtained for 2504 individuals around the world from 1000 Genomes Project database. Populations were broadly classified into five ethnic groups: Americans (AMR), Europeans (EUR), Africans (AFR), East Asians (EAS) and South Asians (SAS). File conversions and manipulations were performed using VCFtools v.0.1.13, PLINK v1.9 and EIGENSOFT v7.2.1. Clustering of populations based on SLC6A4 SNPS The fine population structure of the modern-day humans obtained from 1000 Genomes, based on SLC6A4 SNPs was delineated using Principal Component Analysis (PCA) implemented in PLINK v1.9 using --pca command. The two most informative PCs are discussed and plotted in R v3.5.1. Detection of signature of natural selection on SLC6A4 Cross population extended haplotype homozygosity (XP-EHH) algorithm (http://hgdp.uchicago.edu/Software/) was used for detecting the genetic imprint of recent positive selection by analyzing long-range haplotypes in global human populations obtained from 1000 Genomes. Due to unavailability of updated genetic map information pertaining to the distance between two loci (in centimorgan, $\mathrm{cM})$ and recombination rate for each locus $(\mathrm{cM} / \mathrm{Mb})$ for Human Genome Assembly GRCh38, we could not employ all SNPs for XP-EHH analysis. Out of the 25 diseaserelevant SNPs and all annotated 147 SNPs of SLC6A4, 20 and 38 SNPs respectively could be used for XP-EHH analysis. Identical by Descent (IBD) analysis Populations that have higher IBD sharing for a particular DNA segment can be considered to be under strong and resent selection pressure for that genomic area. Here, IBD sharing was calculated using Pi-Hat statistic implemented in PLINK v.1.9 employing all 147 SNPs of SLC6A4.

\section{Results}

Principal Component Analysis (PCA) PCA performed on all 147 SNPs of SLC6A4 depicted clear and more distinct clustering between Africans (red dots) and the rest of the world along PC1. Additionally, discernible separation among non-Africans was observed along PC2. East Asians (green dots) were found to be present in distinct cluster from other non-African populations. Detection of signature of natural selection on SLC6A4 For disease-relevant SNPs of SLC6A4, long areas of positive XP-EHH scores was observed among the non-African populations suggesting a recent selective sweep. The highest XP-EHH scores were found among East Asians indicating SLC6A4 is under strong selection among people belonging to this ethnic group. Similar to the disease-associated SNPs of SLC6A4, the rest of the SNPs were also found to be under strong selection especially among East Asian populations with significantly high positive XP-EHH scores compared to Africans. Overall, XP-EHH analysis indicated that SLC6A4 has likely undergone a strong selective sweep among the 
East Asians in the recent past. Identical by Descent (IBD) analysis Supporting $\mathrm{XP}-\mathrm{EHH}$ results, the maximum number of individuals with the highest IBD sharing $(\mathrm{Pi}-\mathrm{Hat}=1)$ belong to East Asians $(53.4 \%)$, distantly followed by the native Americans (18.7\%). The IBD analysis corroborated that SLC6A4 has likely undergone a strong recent selective sweep among the East Asians.

\section{Discussion}

In this study we aimed to assess the ancestry specific variation of signatures of natural selection on SLC6A4. To this end, we obtained the whole genome data of 2504 individuals around the world available in 1000 Genomes Project database. We grouped these 2504 individuals into five broad ancestry categories: Native Americans (AMR), Europeans (EUR), Africans (AFR), East Asians (EAS) and South Asians (SAS). We found that SLC6A4 has likely undergone a strong selective sweep among East Asians in the recent past. East Asians also had the highest IBD sharing for SLC6A4, further indicating to be under strong and resent selection pressure for this genomic area. Recently, Lam et al. identified five SNPs in SLC6A4 (rs140700, rs4251417, rs6354, rs25528, rs25531) that are likely associated with depression (Lam et al., 2018). However, likely because of the ancestry of the participants (French), they only found nominally significant association between these variants and depression. Our XP-EHH results indicated minimal selection pressure on populations of European ancestry compared to East Asians. The XP-EHH scores for the candidate SNPs for depression (rs140700, rs4251417, rs6354, rs25528) was found to be significantly higher among East Asians (1.49, 0.61, 1.42 and 1.37 respectively) compared to Europeans $(0.69,-0.17,0.59$ and 0.54 respectively). Our results indicate that the candidate SNPs for depression are under strong evolutionary constraint governed by purifying selection among East Asians. The evolutionary constraint on SLC6A4 is highly ancestry specific and likely appeared after the divergence between East Asians and Europeans $~ 41,000$ years ago. Despite not generating any additional genotyping data, our study can help to extend the current knowledge about the association between SLC6A4 and neurophysiological disorders. 